\title{
Traffic Analysis of a Congested and Uncongested Network in a Sequential Server Model
}

\author{
Afsana Ahamed ${ }^{1}$, Hamid Vakilzadian ${ }^{2}$ \\ ${ }^{1}$ Department of Electrical Engineering, Arkansas Tech University, USA \\ ${ }^{2}$ Department of Electrical and Computer Engineering, University of Nebraska-Lincoln, USA \\ Correspondence: Afsana Ahamed, Department of Electrical Engineering, Arkansas Tech University, USA.
}

\author{
Received: January 13, 2020 \\ Accepted: June 1, 2020 \\ Online Published: June 8, 2020 \\ doi:10.5539/cis.v13n3p1 \\ URL: https://doi.org/10.5539/cis.v13n3p1
}

\begin{abstract}
The efficient flow of messages in a communication network is crucial in today's dynamic environment. These networks are designed to transmit messages in the shortest amount of time. The source sends the messages to the destination through single or multiple servers. Each server contains a buffer that queues the incoming messages when it is busy. This study analyzed the traffic behavior of uncongested and congested wired communication networks for limited and unlimited queue sizes in multiserver configurations with a sequential server model. The performance of the network was measured using three cases: varying the packet length, adding an extra link, and with a broken link connecting the two servers. The performance of the wired communication networks for these three cases was measured based on packet drop rate, throughput, and average end-to-end delay using the OMNeT++ network simulator. Limited and unlimited queue sizes for a sequential server in the network were implemented and simulated to measure the interarrival rate and packet length for optimum performance. For various interarrival rates, the optimum throughput was measured for different queue sizes. The performance with a broken link or the addition of an extra link was assessed and compared with the sequential server model.
\end{abstract}

Keywords: traffic analysis, congested network, uncongested network, communication network, sequential server, end-to-end delay, queuing model, throughput, packet drop rate

\section{Introduction}

The effective, efficient transfer of information requires a quite complex computer communication network with communication infrastructure. This communication infrastructure is invisible to the end user and must be designed and function properly based on user demand. Communication network infrastructure supports high-speed transport mechanisms, such as Ethernet, $\mathrm{Wi}-\mathrm{Fi}^{\circledR}$, routers, etc. It provides advanced technology for a conference meeting, live chats, online games, live television/data streaming, and much more. Due to the demand for more bandwidth and increasing customer needs, current research is concentrated on the design and management of high-performance networks, where design and management decisions are made to reduce congestion and to better manage network resources. Analytical techniques, empirical methodologies, computer simulation, projections based on experience, and experiments are used to evaluate network performance and compare it to other existing state-of-the-art techniques. Design, evolution, and management decisions are made using information on imminent user demand or on the basis of independent predictions in order to develop a system correctly in the shortest amount of time possible.

Congestion in a communcation network degrades the quality of service (QoS), resulting in longer delays, loss of data, and prevention of new incoming data messages (Hemlata \& V.K. Pandey, 2015). In a communication network, the transmission medium can be wired (such as Ethernet cable or optical fiber) or wireless. This medium allows messages or packets to be transmitted between source nodes (or servers) and destination nodes (or servers). The size of the messages varies from bytes to megabytes or gigabytes. To analyze the traffic behavior of these communication networks, different performance parameters are considered, such as packet drop rate, throughput, average end-to-end (E2E) delay, network utilization, and cost. A communication network is said to be in a congested state when the data rate of incoming messages or packets is beyond the capacity of the server. A network can be said to be in an uncongested state when the data rate is lower than the capacity of the servers (Sztrik, 2012). In order to predict the performance of present and future communication networks for resource management, 
various probabilistic models are used. Among them, the network of queues is most important. A study by C. Sutton et al. (Sutton \& Jordan, 2010) combined queueing network models and graphical models to evaluate the performance of communication servers. They used a Markov chain with a Monte Carlo simulation for the prediction of congestion. V. Bhaskar et al. (Bhaskar \& Lallement, 2010) used a network of queues for developing and analyzing the behavior of a supply chain model. Their queuing models helped to decide the average number of products that can be delivered with a minimum response time. They determined the optimum capacity of the queuing network using the least response time. They determined different performance measures, such as average queue lengths, average wait time in the queue, response time, and steady-state probabilities for the network. However, they did not analyze the performance of the queuing network in terms of packet drop rate, throughput, or average end-to-end delay.

Z. Huang (Huang, 2010) developed a simulator that can be run on a parallel computer to find the network's scale invariants and demonstrated that network congestion (dynamical property) depends on network connectivity (topological property). He found that there is an onset relationship between network congestion and network topology. However, Huang's results need to be evaluated for general topologies with different traffic conditions. Mazraani et al. (Mazraani \& Parulkar, 1992) analyzed the behavior of an Ethernet channel in terms of bursty traffic with peak bandwidth, average bandwidth, and burst factor. Their study, however, did not include a critical analysis of system capacity, packet capacity, or interarrival rates. Kadir (Kadir, 2015) modeled the different random entities in an $\mathrm{M} / \mathrm{M} / 1$ queue model using the $\mathrm{MATLAB}^{\circledR}$ simulator to find the similarity with theoretical assumptions. He found that the $\mathrm{M} / \mathrm{M} / 1$ model exactly predicted the distribution relations that are assumed in theoretical derivations. However, network performance metrics were not investigated in terms of various performance parameters, such as queue length, throughput, and interarrival rate. Atefi et al. (Atefi, Yahya, Rezaei, \& Erfanian, 2016) evaluated the M/M/1 queue model in a local area network (LAN). They used Poisson and exponential distributions and compared the traffic behavior of these distributions in terms of some performance metrics, such as average E2E delay, throughput, utilization, and network load. Their results were consistent with the mathematical relations of the $\mathrm{M} / \mathrm{M} / 1$ queue in a LAN. However, the throughput, as well as network utilization and cost, was not analyzed in terms of interarrival rate and queue size.

Mabayoje et al. (Mabayoje, Ameen, Abikoye, Muhammed, \& Olabiyisi, 2011) established an effective, fair queuing model which was capable of decreasing network congestion by allocating network resources among the users. Their proposed model allocated higher priority to real-time applications and achieved better performance parameters, such as throughput and delay. However, the other performance metrics, such as network utilization and packet drop rate, for example, were not examined.

The aforementioned studies did not focus on traffic analysis based on packet length and queue size and with burst traffic packet loss due to the collision, packet delay, and queue size increases (Frost \& Melamed, 1994). Simulation of a small network can help the network analyst to understand the behavior of a large computer network, such as the whole Internet, and to distribute the network resources accordingly on the basis of user demand. The objective of this study was to observe the behavior and the performance characteristics of uncongested and congested data communication queuing models with respect to interarrival rate, queue size, and packet length for optimum throughput. In addition, the impact on network performance of adding or removing a link was investigated. Section 2 is a discussion of communication networks and networks of queues. In Section 3, the experimental setup is introduced, followed by simulation steps to implement, modify, and experiment with the queueing models. In Section 4, simulation results of the traffic behaviors of congested and uncongested sequential communication networks are presented, followed by conclusions in Section 5 .

\section{Communication Networks and Queueing Models}

Computer networks consist of network servers and transmission medium to share data and resources among the nodes that originate, route, and terminate data packets or messages. Simulation of communication networks involves executing a set of tasks simultaneously. These tasks include generation and transmission of packets and queueing them with various queue theories with interarrival-rate and service-rate probabilities. A real-time simulator is required to investigate the real-time traffic scenarios in a communication network. For this purpose, different models were developed in OMNeT+ (Varga, OMNeT++, 2001).

Figure 1 demonstrates a service center with a single server model. When a message arrives at the service center, the server processes the message immediately if the server is free, provided that no messages are waiting in the server. If the server is busy, then the incoming messages are stored in a queue. Those messages wait in the queue until the server becomes available. When the server is available, the message is removed from the queue, processed by the server, and then sent out. The size of the queue depends on the capacity of the service center. 
Once the queue is full, the incoming messages are lost; and the server cannot process them. The single-server model requires two parameters: the arrival rate of messages and the service rate of the server in the service center. For any specific values of queue size, arrival rate, and service rate, the performance evaluation of server utilization (the proportion of time when the server is busy) can be analyzed and the network behavior investigated.

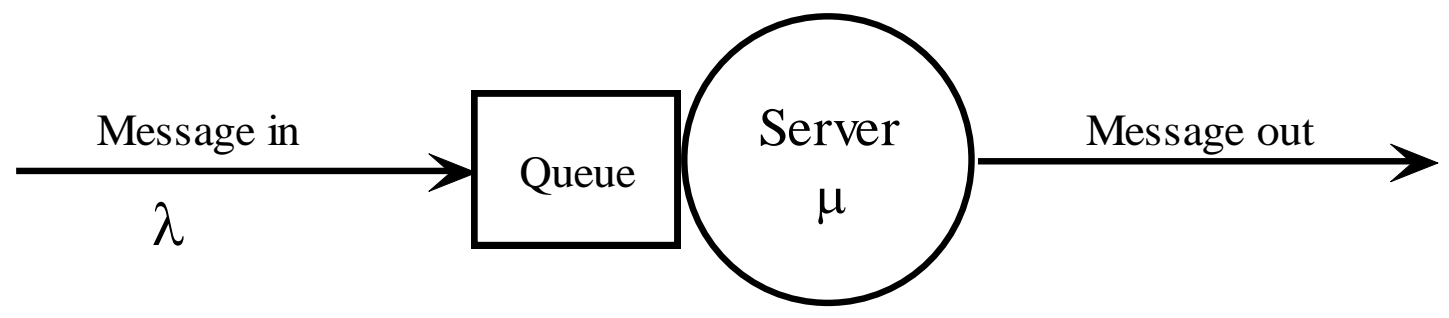

Figure 1. A single-server system

\section{Communication Networks}

Data communication networks are a collection of processors and peripheral devices that exchange data among the user's nodes. Data is transmitted from a source node to a destination node through a wired link(s), fiber optics, or wireless networks. The transmission medium between two end-to-end devices or nodes can have different structures, speeds, and nodes (routers), which can lead to a complicated transmission network. The links can be congested or uncongested to different degrees based on the number of data packet rates, server capacity, and number of end-to-end users, considering urban and metropolitan areas. Congestion may occur as a result of an increase in communication demand, a limited queue size, or a limit in transmission bandwidth (Frost \& Melamed, 1994).

\section{Queueing Models}

In a single $\mathrm{M} / \mathrm{M} / 1$ queuing model, if the server is busy, a packet is stored in a queue when it arrives. The various performance parameters of the queue, such as the long-run average number of messages in the system (L), the average time a packet spends in the queue $\left(w_{Q}\right)$, the long-run average time an arriving packet spends in the system $(\mathrm{w})$, and the time an average number of packets spend in the queue $\left(L_{Q}\right)$ can be calculated using the following relationships (Sztrik, 2012).

$$
\begin{gathered}
\mathrm{L}=\frac{\lambda}{\mu-\lambda} \\
\mathrm{w}=\frac{L}{\lambda} \\
\mathrm{w}_{Q}=\mathrm{w}-\frac{1}{\mu} \\
\mathrm{L}_{Q}=\frac{\lambda^{2}}{\mu(\mu-\lambda)}
\end{gathered}
$$

where, $\mu$ is the service rate, $\lambda$ is the arrival rate, and $\rho$ is the server utilization.

The probability that the system is empty is

$$
P(S=0)=1-\rho
$$

The probability that the queue is empty or there is a packet being processed by the server is 


$$
P(Q=0)=1-\rho+(1-\rho) \rho==1-\rho^{2}
$$

The probability that there are more than $\mathrm{N}$ packets in the system is

$$
P(S>N)=1-\sum_{i=0}^{N}(1-\rho) \rho^{i}=\rho^{N}
$$

The abovementioned relationship provides insight into network performance in terms of network utilization and the probabilistic nature of arrival and service rates. When sequential servers and the network topology are included, they are not able to provide the perception of a complex communication network. Therefore, the relationships are applicable only for single-server model cases and will not work with a sequential server or more complex cases, such as the ones in computer communication networks.

$\$$ LAN
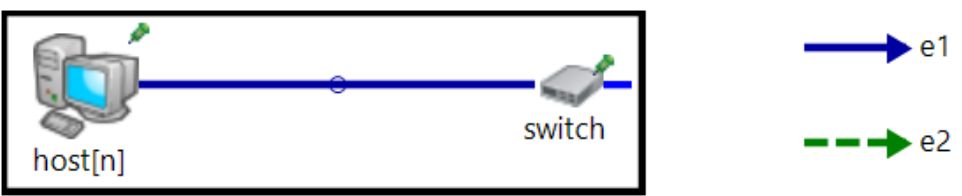

Sequential_server_system

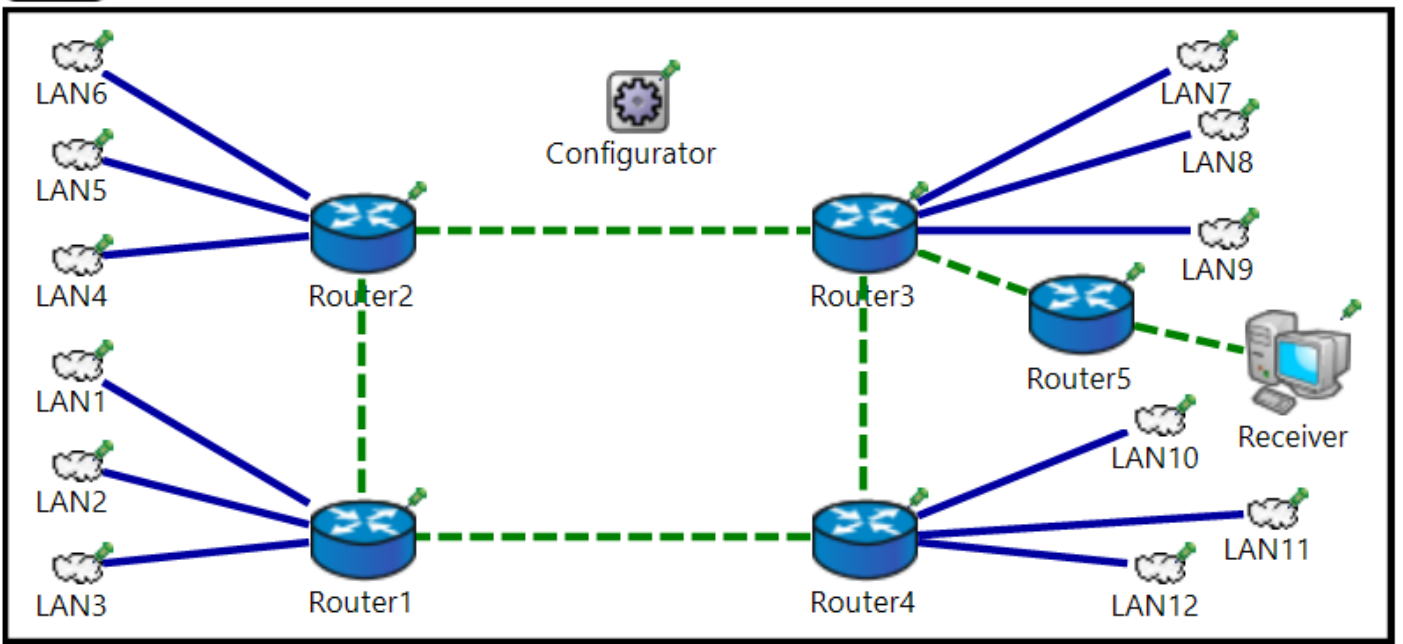

Figure 2. Sequential server system (Ahamed, Sun, Vakilzadian, \& Möller, 2017)

In order to determine the optimum packet size, two models were considered: (1) queue size was not limited $(\mathrm{M} / \mathrm{M} / 1)$, and (2) queue size was limited (M/M/1/K) (Trivedi, 2008). For both cases, the first-in-first-out (FIFO) queue model was employed. For the analysis, we assumed that the system had a drop-tail queue model, where the entering packets or messages fall from the tail end of the queue when it is full. Each packet was treated independently and identically; and when the queue was filled to its maximum capacity or size, the new incoming packets were dropped until the queue had adequate space to accept the new incoming packets or traffic (Santosh Kumar Mishra, 2015). The transmission delays corresponding to the Ethernet lines e1 and e2 in Figure 2 were $100 \mathrm{~ns}$ and $1 \mathrm{~ns}$, respectively; and maximum data rates of 10 Mbps and 100 Mbps were assumed, respectively

For this analysis, we used a sequential server with five routers, as shown in Figure 2. In this configuration, Router 1, Router 2, Router 3, and Router 4 are connected in a ring; and Router 5 is connected in series with 
Router3. Each LAN consists of two hosts connected with a parallel Ethernet switch, shown in Figure 2. Therefore, in this setup, the sequential server model has 24 hosts and 1 receiver that are connected in a star topology. Three different options for analyzing congested and uncongested sequential server topology were considered:
a. Varying packet length
b. Adding an extra link between two routers
c. Removing a link between two routers

For these items, it was assumed that the interarrival rate followed the exponential distribution or uniform distribution and that the service rate followed the Poisson distribution.

In addition, two different alternatives were considered (limited and unlimited queue sizes) for items (a-c) above. The FIFO queue discipline was used for both; and the server had a drop-tail queue model. Two different types of transmission lines were used in this study, including Ethernet 10Base-T, with a processing speed of $10 \mathrm{Mbps}$, and Ethernet 100Base-T, with a processing speed of $100 \mathrm{Mbps}$ (Nikkel, 1997). The service rate, $\mu$, was assumed to be 1220.703 packets per sec and 12207.03 packets per sec with a packet size of 1024 bytes. OMNeT++ (Varga, OMNeT++, 2001) was used in this simulation because it is a public source, flexible, model-based, open architecture simulation environment with powerful graphical user interface support with an embedded simulation kernel.

\section{Results and Analysis}

\section{Case (a): Sequential server models with varying packet length}

For the sequential server model in Figure 2, the packet drop rate, average E2E delay, and throughput were studied using exponential distribution for $\lambda$. Both unlimited queue size and queue sizes with 5,10 , and 50 packets with a packet interarrival rate, $\lambda$, of 500 packets per sec were used.

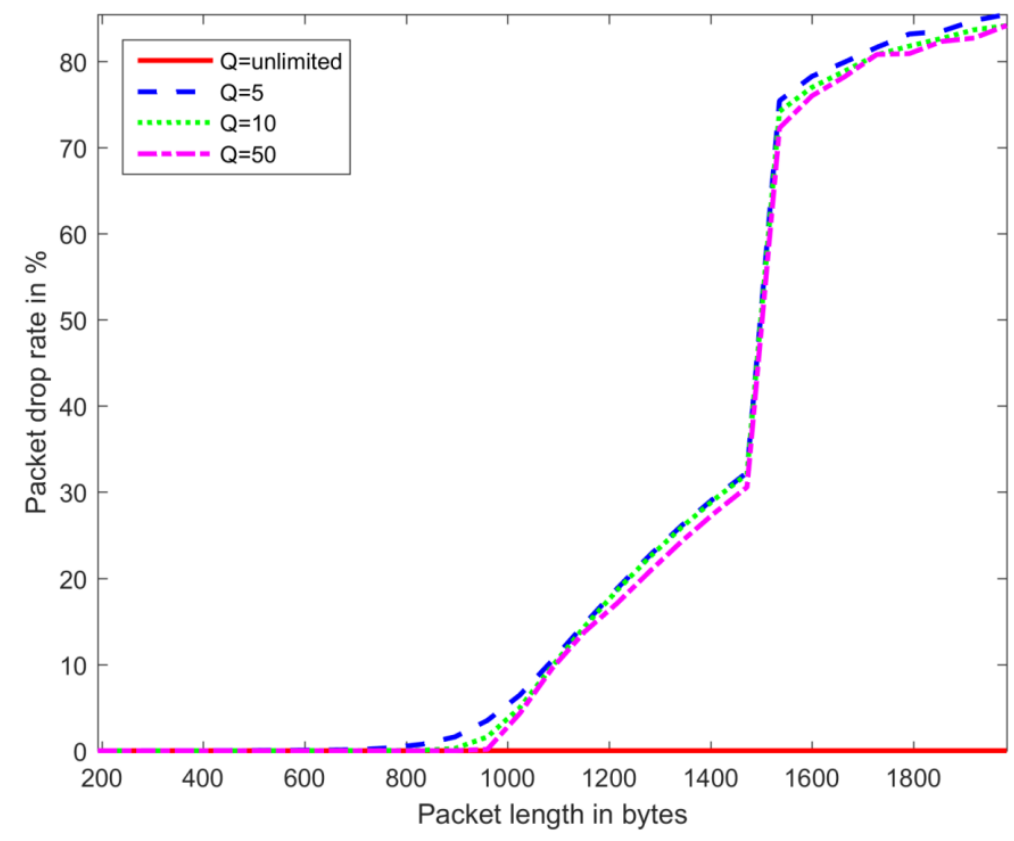

Figure 3. Percentage of packet drop vs. packet length

Figure 3 shows the percentage of packet drop rate versus the arrival rate for congested and uncongested networks for a sequential server queue model. As shown, the increase in packet length caused an increase in the packet drop rate for limited size queues. However, it remained zero for an unlimited size queue.

For packet lengths below 800 bytes, the packet drop rate was low, which agreed with the expectation that when the arrival rate, $\lambda$, is small compared to the service rate, $\mu$, not many packets will drop. With an increase in packet length, the server won't be able to serve all of the received packets; therefore, the packet drop rate goes 
up. The packet drop rates remained almost the same for limited queue sizes of 5,10 , and 50 packets when $\lambda>\mu$. Moreover, the network became congested when the packet length was greater than 256 bytes (from the numerical simulation results) with $\lambda=500$ packets per sec. As shown in Figure 3, there was a sharp rise in the drop rate after a packet length of 1472 bytes. This is because the switch and Router 3 had lower service rates compared to the interarrival rate; and server utilization, $\rho$, was larger than 21, as shown in Tables 1 and 2 for the switch and Router 3, respectively.

Table I. Utilization of the switch

\begin{tabular}{|c|c|c|c|c|}
\hline $\begin{array}{c}\text { Service Rate, } \boldsymbol{\mu} \\
(\text { Mbps })\end{array}$ & $\begin{array}{c}\text { Packet Size } \\
\text { (Bytes) }\end{array}$ & $\begin{array}{c}\text { Arrival rate, } \boldsymbol{\lambda} \\
\text { (Packets per Sec) }\end{array}$ & $\begin{array}{c}\text { Number of } \\
\text { Hosts, } \mathbf{n}\end{array}$ & Server Utilization, $\boldsymbol{\rho}$ \\
\hline 10 & 1472 & 500 & 2 & 1.17760 \\
\hline 10 & 1472 & 550 & 2 & 1.29536 \\
\hline 10 & 1536 & 500 & 2 & 1.22880 \\
\hline 10 & 1536 & 550 & 2 & 1.35168 \\
\hline
\end{tabular}

Table II. Utilization of Router 3

\begin{tabular}{|c|c|c|c|c|}
\hline $\begin{array}{c}\text { Service Rate, } \boldsymbol{\mu} \\
\text { (Mbps) }\end{array}$ & $\begin{array}{c}\text { Packet Size } \\
\text { (Bytes) }\end{array}$ & $\begin{array}{c}\text { Arrival Rate, } \boldsymbol{\lambda} \\
\text { (Packets per Sec) }\end{array}$ & $\begin{array}{c}\text { Number of } \\
\text { hosts, } \mathbf{n}\end{array}$ & Utilization, $\boldsymbol{\rho}$ \\
\hline 100 & 1472 & 500 & 24 & 1.4131 \\
\hline 100 & 1472 & 550 & 24 & 1.5544 \\
\hline 100 & 1536 & 500 & 24 & 1.4746 \\
\hline 100 & 1536 & 550 & 24 & 1.6220 \\
\hline
\end{tabular}
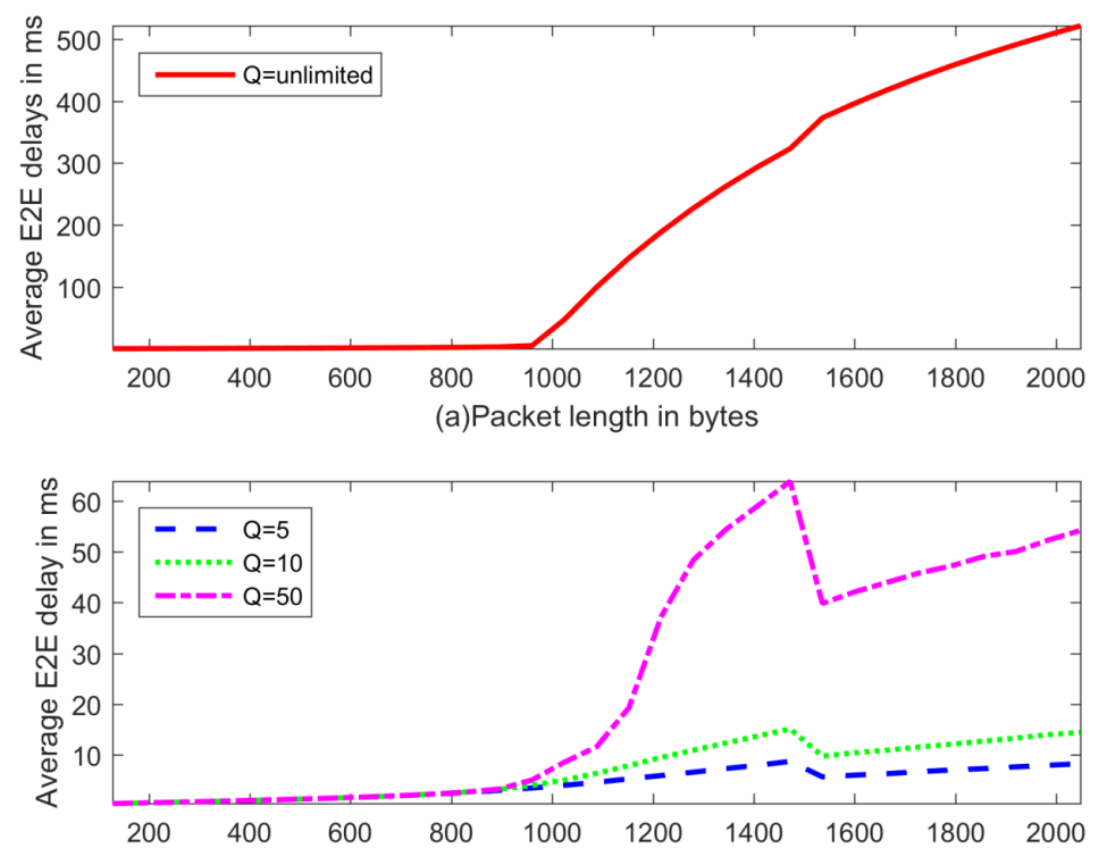

(b) Packet length in bytes

Figure 4. Average E2E delay vs. packet length

The probability that there would be more than one packet in the queue for Router 1 was 0.0312 (8). The long-run average number of packets in the system, L, was 0.2145 (1). 
Figure 4 shows the average E2E delay versus the packet length with $\lambda=500$ packets per sec. The delay increased as the packet length increased. The delay in an unlimited queue was higher compared to the average end-to-end delay in a limited queue size model case. In an unlimited queue size model, more packets waited in the queue for processing, which increased the queueing delay and led to an increase in the average E2E delay.

For a packet length of 1024 bytes, the average increase in E2E delay was $25.3 \%$ when the capacity of the queue changed from 5 to 10 packets and $115.4 \%$ when the queue size changed from 5 to 50 . It was observed that there was a sharp drop in average E2E delays after the packet length $=1472$ packets since the switch and router dropped most of the packets. As a result, the average wait time in the queue decreased.

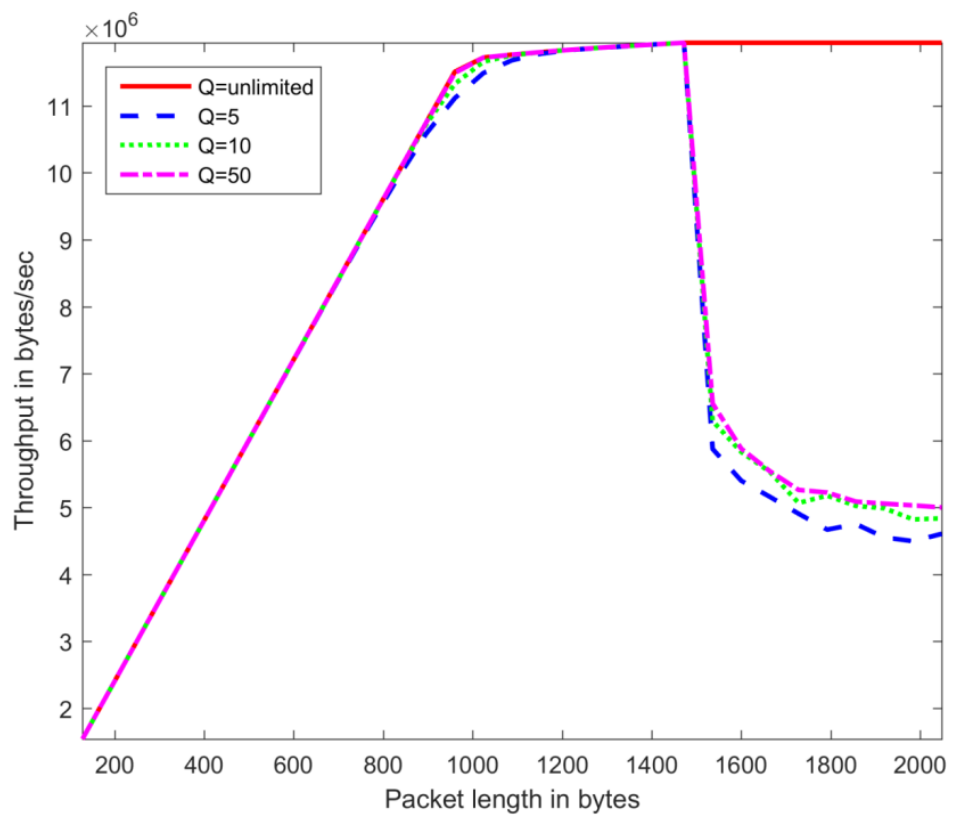

Figure 5. Throughput vs. packet length

Figure 5 depicts the throughput versus packet size for the unlimited queue size and queue sizes of 5, 10, and 50 packets again. For these cases, throughput saturated when the interarrival rate was larger than the service rate $(\lambda>$ $\mu$ ). The critical values of packet length for a queue size of 5,10 , and 50 packets were higher than the critical value of a packet length for the unlimited queue size. The values for queue sizes of 5, 10, and 50 packets were 1088 bytes, 832 bytes, and 896 bytes, respectively (Figure 6). Again, there was a sharp drop in throughput after packet lengths of 1472 bytes for the limited size queues.

Due to a one-to-one relationship of network utilization and cost with throughput, these results have not been depicted here. The network cost depended on the network utilization; the more time the network was busy, the higher the cost for transmission of packets.

The impact of queue size on the packet drop rate is shown in Figure 6 for interarrival rates, $\lambda$, of 500 and 550 packets per second and packet lengths of 1472 and 1536 bytes. As can be seen in Figure 6, when the capacity of the queue increased, the packet drop rate went down because more packets could be queued for future processing.

For the arrival rate of 500 packets per sec with a packet length of 1472 bytes and a queue size of 50 , the packet drop rate was $30.57 \%$. However, it increased by $6.48 \%$ when $\lambda$ increased to 550 packets with the same size packet length. The packet losses increased by $41.7 \%$ and $49.54 \%$ for $\lambda=500$ and 550 packets with packet lengths of 1472 bytes and 1536 bytes, respectively. 


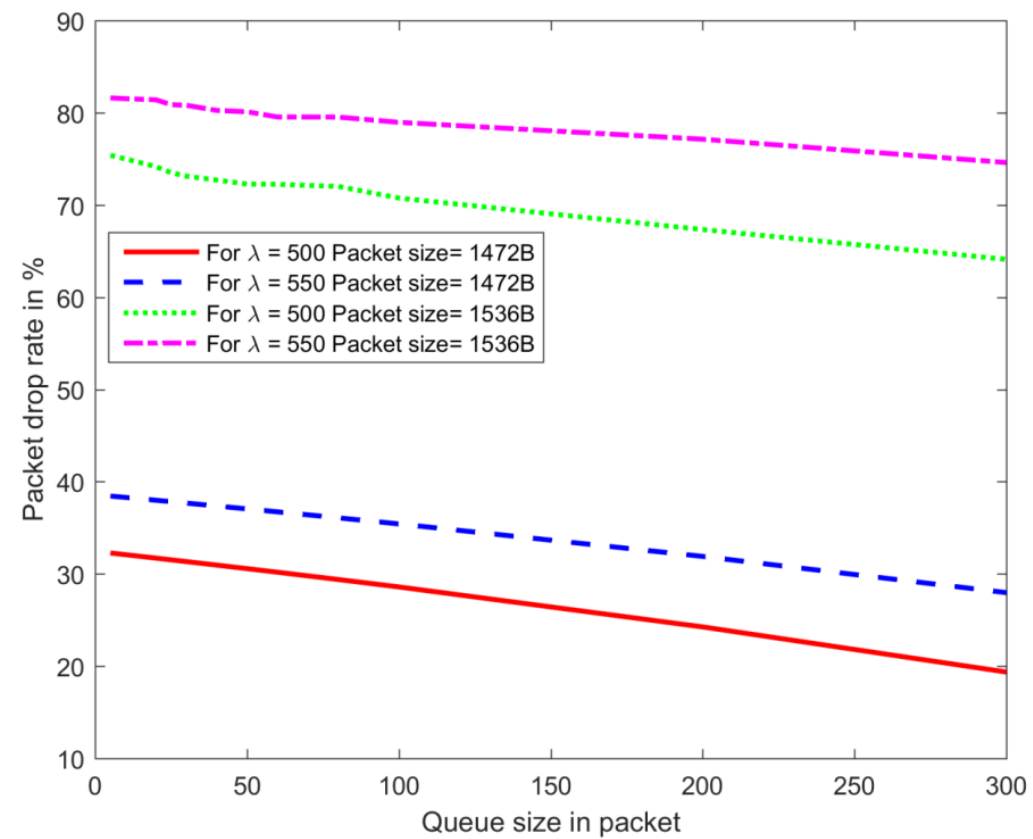

Figure 6. Percentage of packet drop vs. queue size

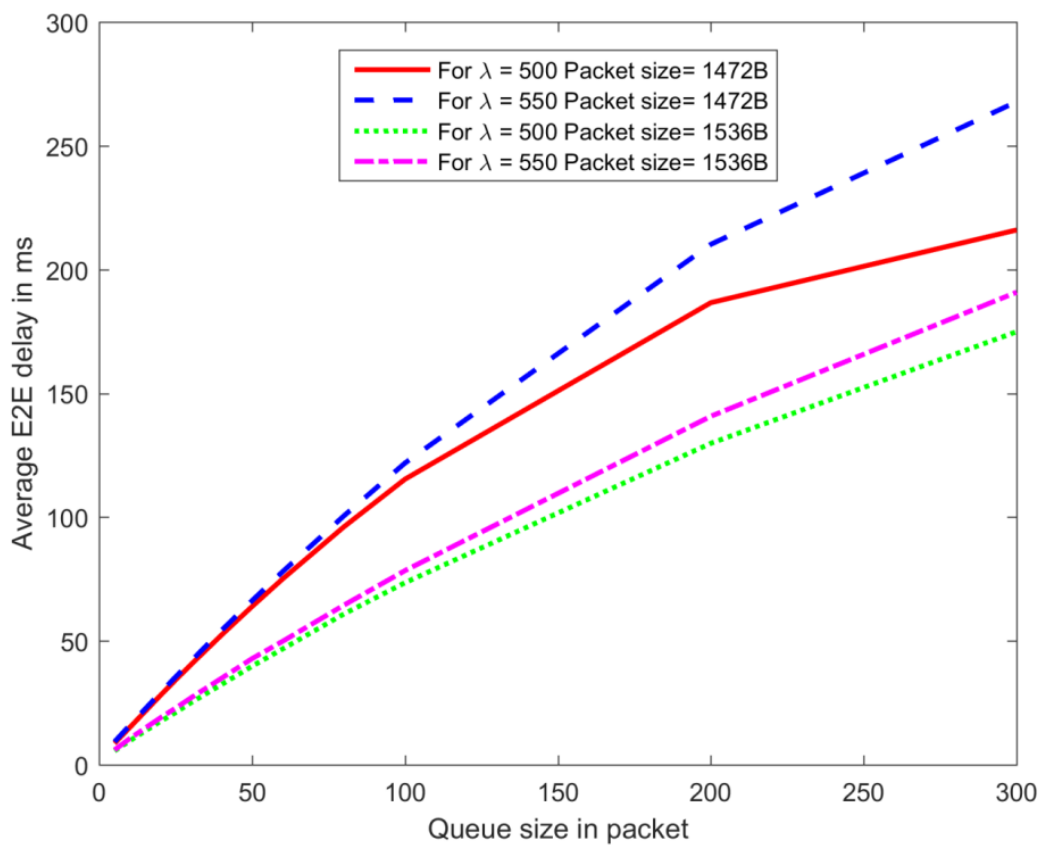

Figure 7. Average E2E delay vs. queue size 


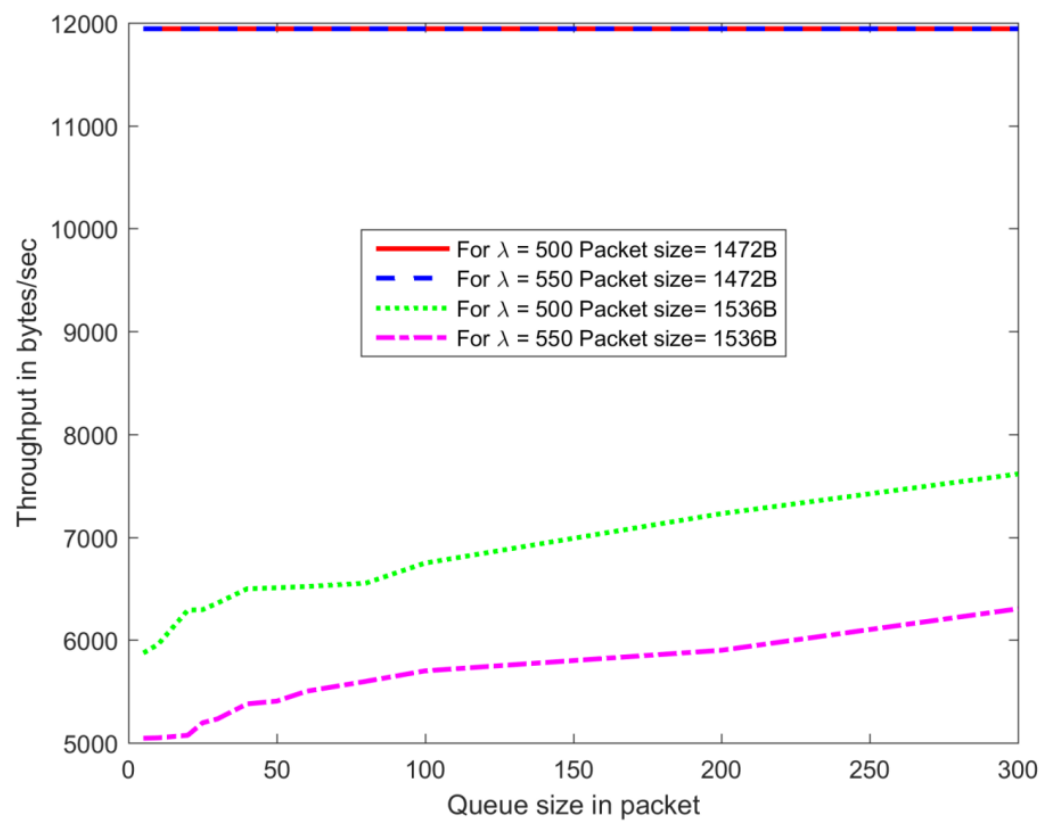

Figure 8. Throughput vs. queue size

The average E2E delays for different values of $\lambda$ (500 and 550) and packet lengths of 1472 bytes and 1536 bytes are shown in Figure 7. For a queue size of 50 packets, the average increase in E2E delay was $2.36 \mathrm{~ms}$ when $\lambda$ increased from 500 to 550 packets per sec with the same packet size. However, when the packet size increased from $1472 \mathrm{~B}$ to $1536 \mathrm{~B}$, the E2E delay decreased by $24.11 \mathrm{~ms}$ with the same values of $\lambda=500$ packets per sec. When both $\lambda$ and packet size increased, a $21.04 \mathrm{~ms}$ decrease was observed in the average E2E delay.

Figure 8 presents the network throughput versus the queue size for four different values of $\lambda$ and packet sizes. The critical value of the queue size occurred when the throughput became saturated. This study found that the critical size of the queue when $\lambda$ was 500 and 550 packets per second with a packet size of 1472B was 5 . However, for a packet size of 1536 bytes, the throughput increased with the capacity of the queue due to the decreased service rate for the switches and Router 3. Therefore, the critical value of the packet length for optimum throughput of 1472 bytes was obtained.

\section{Case b): Addition of an extra link}

In this case, the performance metrics of the sequential server model were studied when a link was added between Router 1 and Router 3 in Figure 2.

The additional link connected between Router 1 and Router 3 had a data rate of 100 Mbps. For this model, the packet drop rate, average E2E delay, and throughput for both Poisson and uniform distributions for unlimited queue size and queue sizes of 5, 10, and 50 packets were studied. Here, a packet length of 1024 bytes was considered.

Figure 9 shows the packet drop rate vs. interarrival rate for an unlimited queue size as well as queue sizes of 5, 10, and 50. For this queue size, there was no packet drop from the queue when the arrival rate was less than the service rate $(\lambda<\mu)$. This means that a large number of messages could be transmitted through the link in a short period; and as long as $\mu<\lambda$, those packets can wait in the unlimited queue for future processing. For limited queue size cases with $\lambda<\mu$, the system was uncongested. However, when $\lambda$ was increased above $\mu$, the packet drop rate increased; and the network became congested. For $\lambda=500$ packets per second, when the queue size increased from 5 to 10 and 5 to 50 , the packet drop rate decreased by $1.64 \%$ and $2.33 \%$, respectively (Figure 9). 


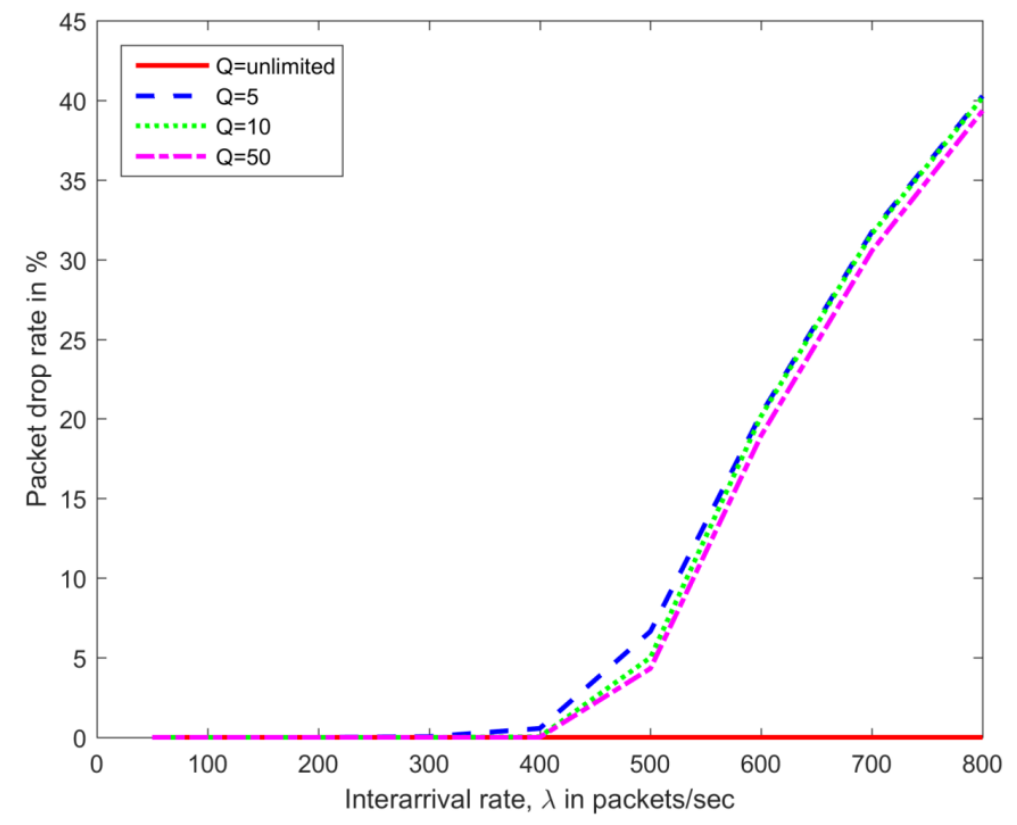

Figure 9. Percentage of packet drop vs. interarrival rate

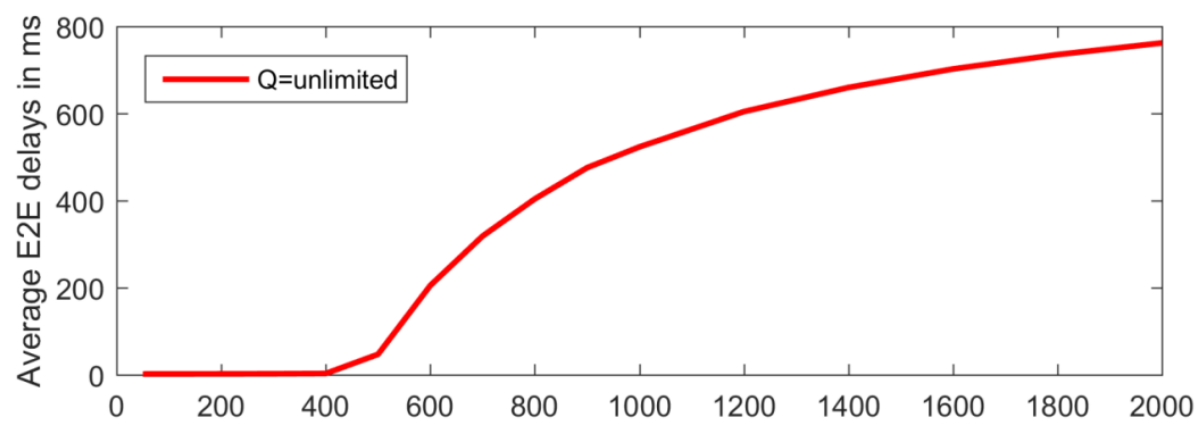

(a) Interarrival rate, $\lambda$ in packets/sec

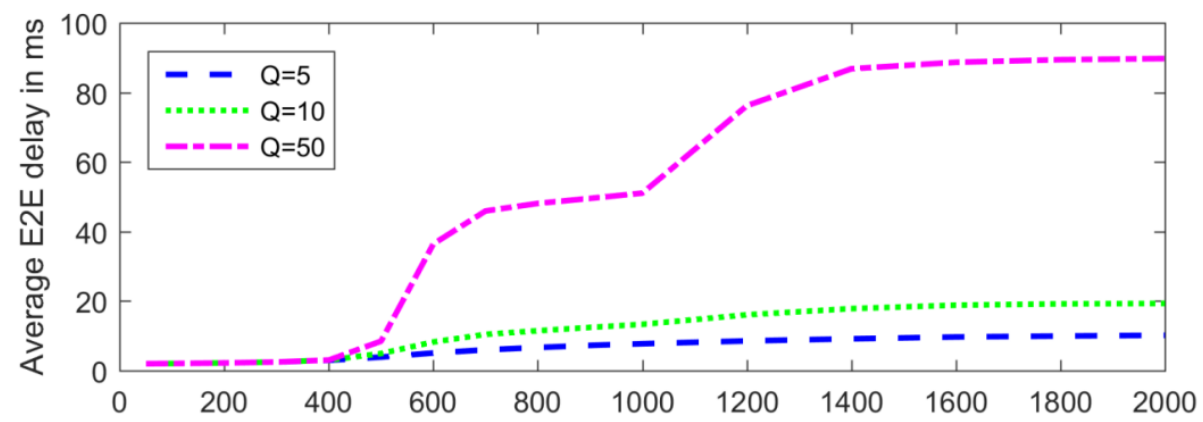

(b) Interarrival rate, $\lambda$ in packets/sec

Figure 10. Average E2E delay vs. interarrival rate

Figure 10 (a) depicts the average E2E delay versus the interarrival rate for unlimited queue size, and 12(b) shows the same delay for queue sizes of 5, 10, and 50 packets. As seen in Figure 12 (a), the average E2E delay increased with an increase in the interarrival rate, since more packets can wait in the queue for future processing. As a result, the average E2E delay was high. For the interarrival rate, $\lambda$, of 500 packets per sec, the average E2E 
delay increase was $26.23 \%$ and $116.5 \%$, when the capacity of the queue changed from 5 to 10 and 5 to 50 packets, respectively.

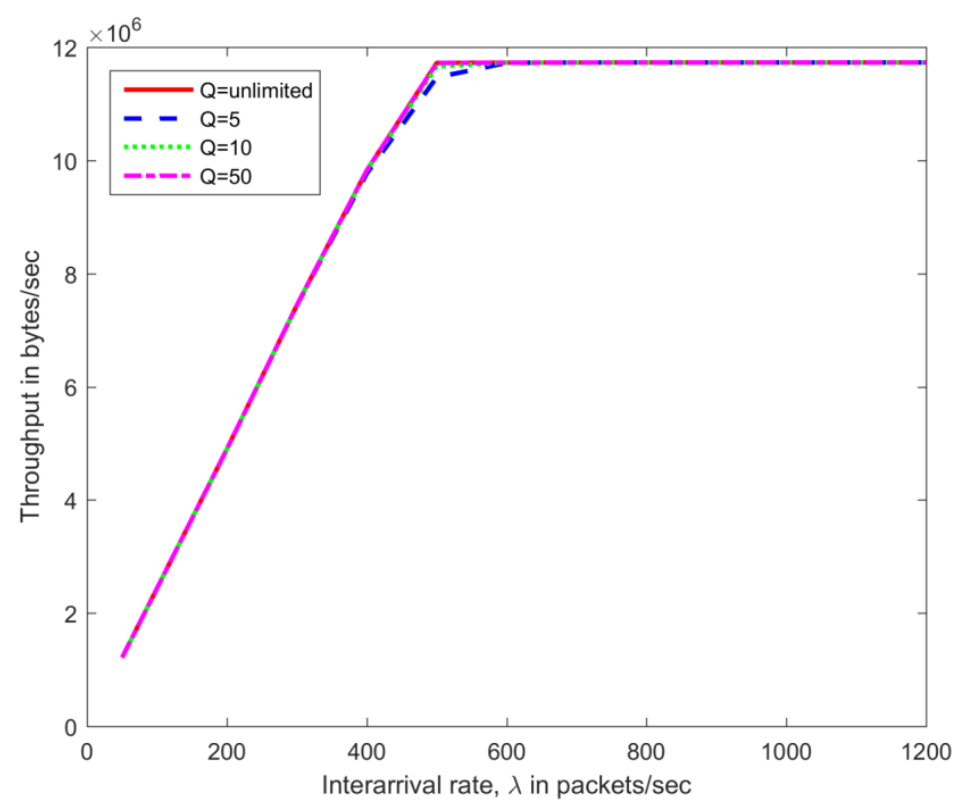

Figure 11. Throughput vs. interarrival rate

Figure 11 represents the throughput versus interarrival rate. For all four cases, the throughput increased linearly with $\lambda$ when $\lambda<500$; and it became saturated when $\lambda>\mu$, which happened when the service rate, $\mu$, went above 588 packets/sec. The network utilization and network cost for the sequential server system had similar results.

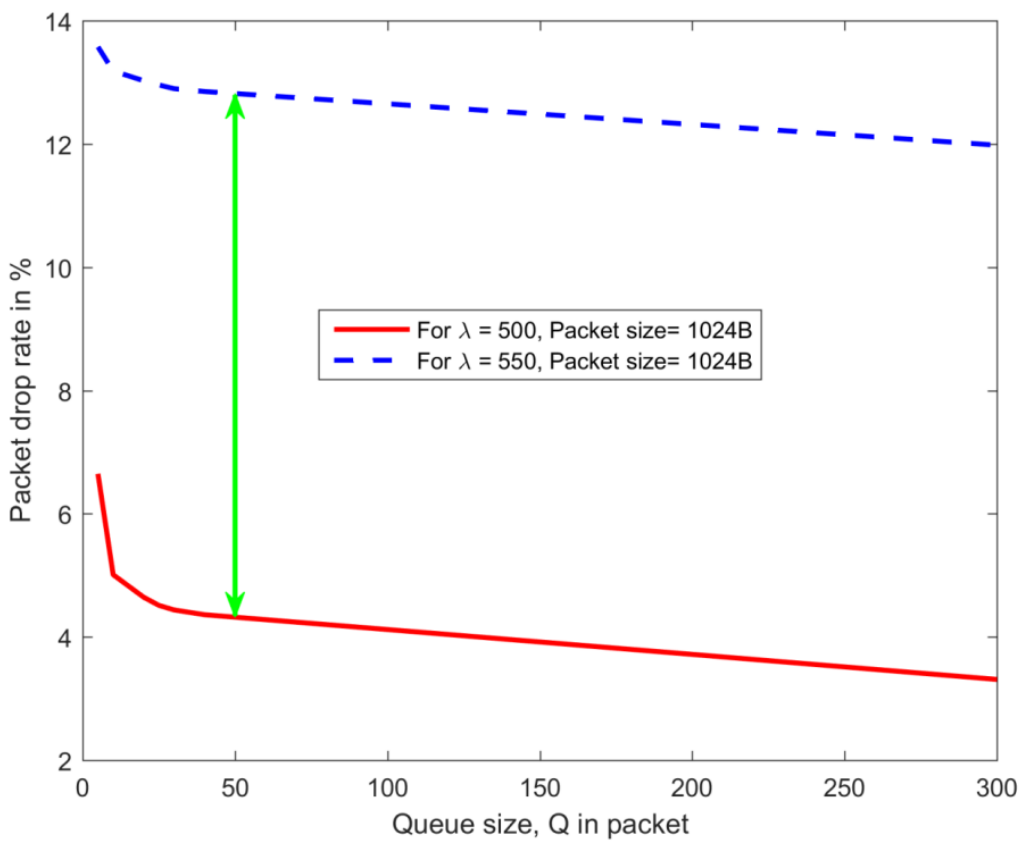

Figure 12. Packet drop rate in $\%$ vs. queue size 


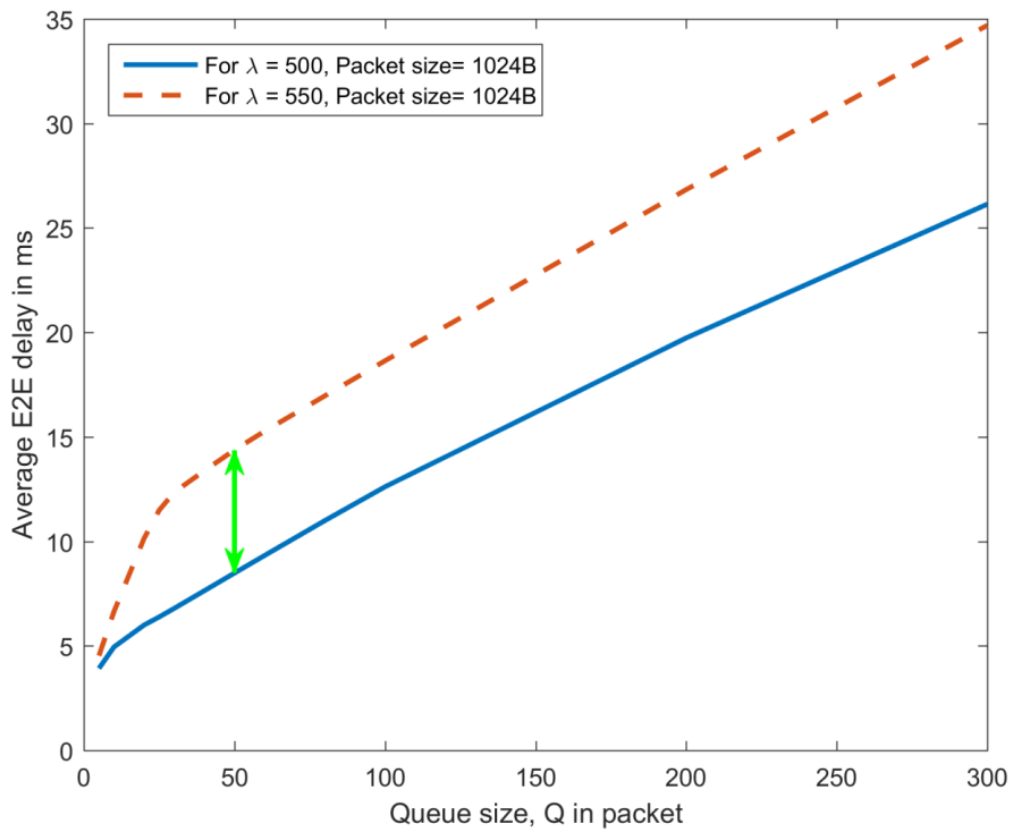

Figure 13. Average E2E delay vs. queue size

Figure 12 shows the change in packet drop rates vs. the queue size for the sequential model for the packet arrival rates of 500 and 550 per second and packet size of 1024 bytes. In this case, the packet drop rate decreased as the queue size increased. For a queue size of 50, the packet drop rate decreased by $8.5 \%$ when $\lambda$ increased from 500 to 550 packets/sec. The average E2E delays for two different values of $\lambda$ are provided in Figure 13. Here, the average E2E delays increased with the increase in queue size. For a queue size of 50, the average E2E delay increased by $8.5 \mathrm{~ms}$ with an increase in $\lambda$ from 500 to 550 packets per sec.

Figure 14 shows throughput vs. queue size for $\lambda$ of 500 and 550 packets/sec. The throughput became saturated when queue capacities were above 10 and 40 for interarrival rates of 500 and 550, respectively. Saturation occurred since the drop rate depended only on the service rate. Since the overall service rate (588 packets/sec from Figure 13) of this sequential server was greater than 500, saturation occurred early when $\lambda$ was 500 , as compared to $\lambda=550$.

In the second part of Case (b), this model, when $\lambda$ followed the uniform distribution, was analyzed. Here, cases similar to Poisson distribution were considered (unlimited queue and queues sizes of 5, 10, and 50 packets). The results for unlimited queue size are shown in Table III. The packet drop rate for unlimited queue size for all of the interarrival rates was zero. However, the system had a high average delay. In Figure 15, the packet drop rate and average E2E delays vs. interarrival rate are depicted for queue sizes of 5,10, and 50 packets, respectively. It can be seen that the packet drop rate decreased as the interarrival rate decreased. They remained the same for all queue sizes. Nevertheless, the average E2E delay increased with increased queue sizes.

Table III. Sequential server model with unlimited queue size for uniform distribution

\begin{tabular}{|c|c|c|}
\hline Interarrival Rate, $\boldsymbol{\lambda}$ & Packet Drop Rate (\%) & Average E2E Delay (ms) \\
\hline $200-1000$ & 0.000 & 002.202 \\
\hline $1000-2000$ & 0.000 & 642.699 \\
\hline $2000-10000$ & 0.000 & 857.512 \\
\hline $10000-20000$ & 0.000 & 965.217 \\
\hline
\end{tabular}




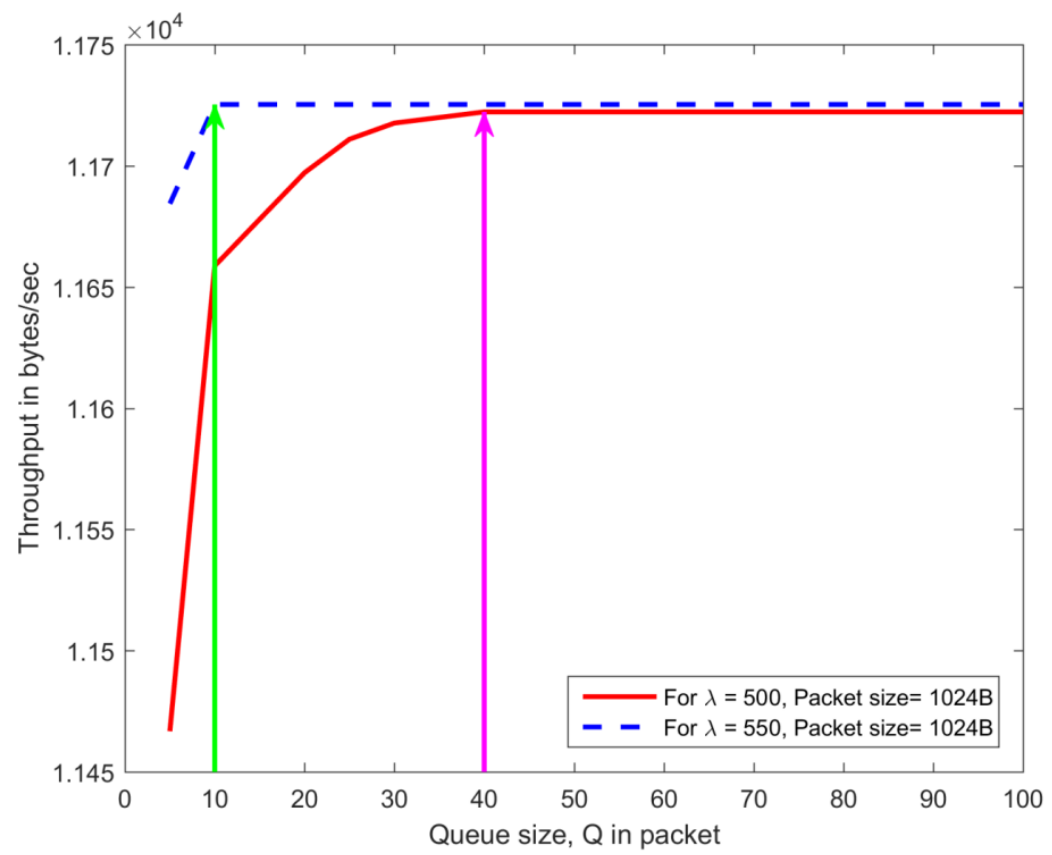

Figure 14. Throughput vs. queue size

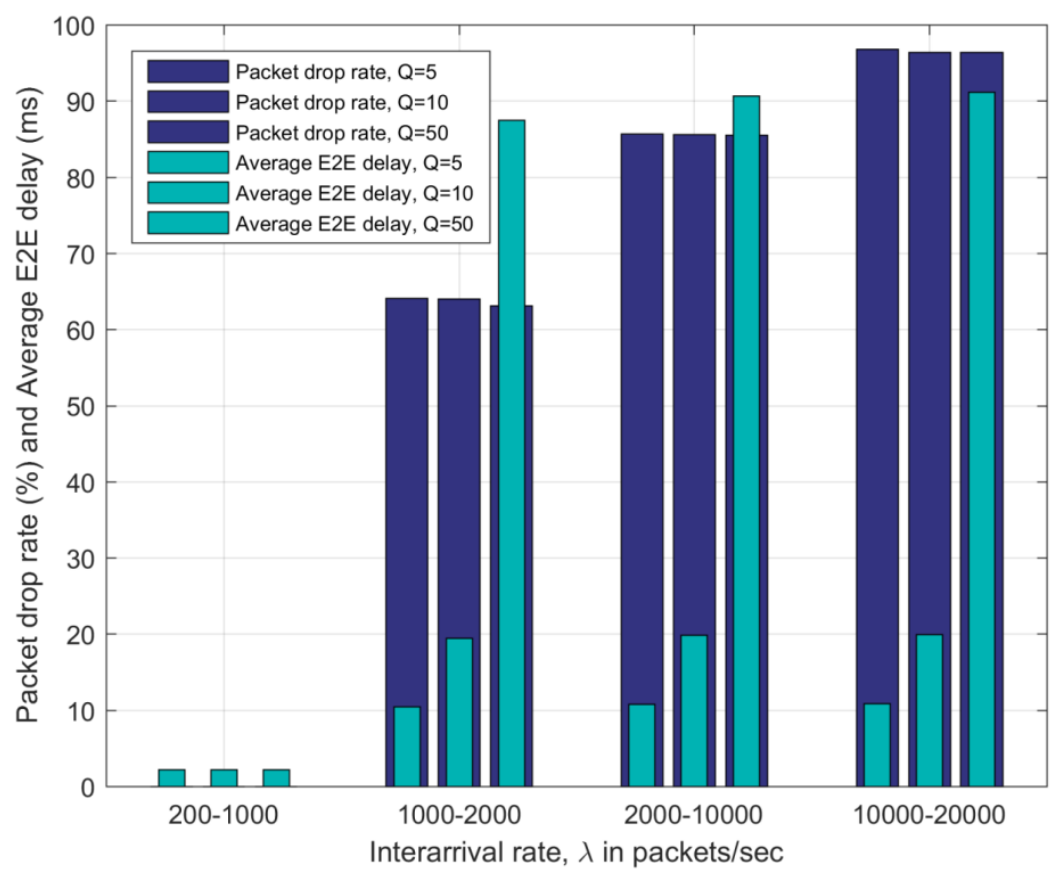

Figure 15. Packet drop rate and average E2E delays vs. interarrival rate

For this model, the packet drop rate again averaged E2E delay, and the throughput for both exponential and uniform distributions (for $\lambda$ ) were analyzed. It was found that the results were similar to the previous cases and packet size.

\section{Case (c): A broken link for sequential server model}

In this case, the sequential server model with a broken link between Router 1 and Router 2 was considered; and the performance metrics were observed and analyzed for unlimited and limited queue sizes of 5, 10, and 50 
packets. Two different distributions of the interarrival rate were also measured. For Poisson distribution of $\lambda$, the resultant graphs of packet drop rate, average E2E delay, and throughput were similar to Case(b). Due to the broken link, the packet drop rates and the average E2E delays were slightly higher; the throughput was slightly lower, as compared to the Case(b) results shown in Figures 9-11.

The observation on the queue size also had the same results as shown in Figures 12-14 with a little bit of change due to the broken link. Here, the throughput increased linearly with queue size and saturated when the queue size became larger than the critical value.

The results of packet drop rate and E2E delay for various interarrival rates and unlimited queue size using uniform distribution were the same as those shown in Table III. Also, the changes in packet drop rate and average E2E delays vs. interarrival rate for uniform distribution for different queue sizes were similar to Figure 15. In other words, change in the distribution function (uniform distribution or Poisson distribution) did not make any difference in the results.

Table IV is a comparison of the E2E delay and packet drop rate for Case (b) when a link was added and Case (c) when a link was removed. Adding an extra link reduced the packet drop rate and service rate (Ahamed, Sun, Vakilzadian, \& Möller, 2017); however, it increased the average E2E delays, requiring less queue size. The performance and behavior of the sequential server queuing models in this study were consistent with (Huang, 2010), (Kadir, 2015), and (Atefi, Yahya, Rezaei, \& Erfanian, 2016).

Table IV. Comparison of sequential server model with different scenarios for $\lambda=500$ and a queue size of 10

\begin{tabular}{|l|c|c|c|c|c|}
\hline \multicolumn{1}{|c|}{ Methods } & $\begin{array}{c}\text { Packet Drop } \\
\text { Rate (\%) }\end{array}$ & $\begin{array}{c}\text { Average E2E } \\
\text { Delay }(\mathbf{m s})\end{array}$ & $\begin{array}{c}\text { Empirical Values } \\
\text { of Service Rate, } \boldsymbol{\mu}\end{array}$ & $\begin{array}{c}\text { Critical Value } \\
\text { of Queue }\end{array}$ & $\begin{array}{c}\text { Critical Value of } \\
\text { Throughput }\end{array}$ \\
\hline Case (b) & 5.01001168 & 4.94673 & 588 & 70 & 11722240 \\
\hline $\begin{array}{l}\text { (Ahamed, } \\
\text { Vakilzadian, \& Möller, } \\
\text { 2017) }\end{array}$ & 5.173706573 & 5.03049 & 544 & 60 & 11722240 \\
\hline Case (c) & 4.321117 & 8.51035 & 513 & 40 & 11722240 \\
\hline
\end{tabular}

\section{Conclusions}

This paper presents a study of the traffic behavior of uncongested and congested communication networks for sequential server queueing models using three different cases: varying packet length, adding an extra link, and dropping a link to account for a broken link. Poisson and uniform distributions were used to simulate the arrival rates of the message. Unlimited and limited queue sizes were considered when observing the behavior of these models, and critical values of the queue size were measured for better resource management. The packet drop rate, average E2E delay, network utilization, throughput, and cost for the sequential models in these three different scenarios were observed using a real-time simulator. It was found that the traffic behavior resembled the theoretical model of the queuing system as expected. The critical values were also obtained for the size of the queue and interarrival rate when the throughput was saturated for these sequential server models. The critical value for packet length was obtained from Case (c) where the throughput was optimum. The unlimited queue size model had a zero drop rate, higher E2E delay, higher throughput, higher utilization, and higher network cost than the queue model with a certain queue size in all of the cases. However, throughput became saturated when the queue size and interarrival rate were beyond the critical point for all cases. The performance of Case (b) and Case (c) were compared (Ahamed, Sun, Vakilzadian, \& Möller, 2017), and Case (b) had better performance as compared to other scenarios in terms of packet drop rate and queue size. However, in Case (c), the average E2E delay was higher.

\section{References}

Ahamed, A., Sun, H., Vakilzadian, H., \& Möller, D. P. (2017). Performance analysis of a congested and uncongested communication network. IEEE International Conference on Electro Information Technology (pp. 557-563). Lincoln, Nebraska. https://doi.org/10.1109/EIT.2017.8053427 
Atefi, K., Yahya, S., Rezaei, A., \& Erfanian, A. (2016). Traffic behavior of Local Area Network based on $\mathrm{M} / \mathrm{M} / 1$ queuing model using poisson and exponential distribution. Region 10 Symposium (TENSYMP), 2016 IEEE, (pp. 19-23). https://doi.org/10.1109/TENCONSpring.2016.7519371

Bhaskar, V., \& Lallement, P. (2010). Modeling a supply chain using a network of queues. Applied Mathematical Modelling, 34, 2074-2088. https://doi.org/10.1016/j.apm.2009.10.019

Frost, V. S., \& Melamed, B. (1994, March). Traffic modeling for telecommunications networks. IEEE Communications Magazine, 32, 70-81. https://doi.org/10.1109/35.267444

Hemlata, \& V.K. Pandey. (2015). Automatic Traffic Management and Congestion Avoidance. International Journals of Advanced Research and Management.

Huang, Z. (2010). Topology and congestion invariant in global internet-scale networks. Ph.D. dissertation.

IEEE 802.3. (2017, Sep). Wikimedia Foundation. Retrieved from https://en.wikipedia.org/wiki/IEEE_802.3

Kadir, N. (2015). Observation of the common phenomena from a MATLAB model for M/M/1 queuing system. Advances in Electrical Engineering (ICAEE), 2015 International Conference on, (pp. 210-212). https://doi.org/10.1109/ICAEE.2015.7506833

Mabayoje, M. A., Ameen, A. O., Abikoye, O. C., Muhammed, R., \& Olabiyisi, S. O. (2011). An efficient fair queuing model for data communication networks. International Journal of Computer Science and Information Security, 9, 206.

Mazraani, T. Y., \& Parulkar, G. M. (1992, Dec). Performance analysis of the Ethernet under conditions of bursty traffic. Global Telecommunications Conference, 1992. Conference Record., GLOBECOM '92. Communication for Global Users., IEEE, (pp. 592-596 vol.1). https://doi.org/10.1109/GLOCOM.1992.276446

Nikkel, S. (1997). Network connection speeds reference.

Santosh Kumar Mishra, B. A. (2015). Analysis of the Performance of Drop Tail-Aqm Techniques. International Journal of Research Review in Engineering and Technology, 3(4).

Sutton, C. A., \& Jordan, M. I. (2010). Inference and learning in networks of queues. International Conference on Artificial Intelligence and Statistics, (pp. 796-803).

Sztrik, J. (2012). Basic Queuing Theory. University of Debrecen, Faculty of Informatics.

Trivedi, K. S. (2008). Probability \& statistics with reliability, queuing and computer science applications. John Wiley \& Sons.

Varga, A. (2001). OMNeT++. Retrieved September 7, 2017, from https://omnetpp.org/

Varga, A., \& Hornig, R. (2008). An Overview of the OMNeT++ Simulation Environment. Proceedings of the 1st International Conference on Simulation Tools and Techniques for Communications, Networks and Systems $\backslash \&$ Workshops (pp. 60:1--60:10). ICST, Brussels, Belgium, Belgium: ICST (Institute for Computer Sciences, Social-Informatics and Telecommunications Engineering).

https://doi.org/10.4108/ICST.SIMUTOOLS2008.3027

\section{Copyrights}

Copyright for this article is retained by the author(s), with first publication rights granted to the journal.

This is an open-access article distributed under the terms and conditions of the Creative Commons Attribution license (http://creativecommons.org/licenses/by/4.0/). 